\title{
Alternating perceptions of ambiguous scenes: what's out there? John Rinzel
}

Address: Center for Neural Science and Courant Institute of Mathematical Sciences, New York University, NY, USA

Email: John Rinzel - rinzel@cns.nyu.edu

from Seventeenth Annual Computational Neuroscience Meeting: CNS*2008

Portland, OR, USA. 19-24 July 2008

Published: II July 2008

BMC Neuroscience 2008, 9(SuppI I):LI doi:I0.1 I86/I47I-2202-9-SI-LI

This abstract is available from: http://www.biomedcentral.com/I47I-2202/9/SI/LI

(c) 2008 Rinzel; licensee BioMed Central Ltd.

When visualizing an ambiguous scene (such as the Necker cube) one may perceive ongoing random alternations between the possible interpretations. Dynamical models implement competition as reciprocal inhibition between neuronal populations; dominance alternates while slow negative feedback, adaptation, sets the basic time scale (seconds) for switching. When adaptation is strong enough it overcomes dominance and alternations occur intrinsically and periodically; noise perturbs the regularity. In a different framework, with attractor-based dynamics, adaptation is weak and switches are induced by noise operating on a bistable system. We find that statistics of the observed alternations provide constraints that favor an operating range near the transition zone between the parameter regimes for the two mechanisms. In some paradigms one can manipulate stimulus cues to bias the competition away from equal dominance. We have proposed that the percentage of time dominant is a measure for the likelihood of valid interpretation of the scene. 